\title{
TEST IMMUNOENZIMATICO PER GDH IN CLOSTRIDIUM DIFFICILE: PRIMA O DOPO DEL TEST PER LATOSSINA?
}

\author{
Solaro M., Urbani G., Colombo L., Mocarelli P. \\ Servizio Universitario di Medicina di Laboratorio \\ Ospedale di Desio, Desio (MI)
}

\section{Introduzione.}

La diarrea associata ad antibiotici (DAA) è causata nel 25\% dei casi da Clostridium difficile, agente eziologico della maggior parte delle coliti pseudomembranose. La patologia è sostenuta dalle tossine A e B prodotte dai ceppi tossigenici. I test immunoenzimatici per le tossine $\mathrm{A}$ e/o $\mathrm{B}$ raggiungono una sensibilità massima dell' $80 \%$. I risultati dovrebbero essere disponibili tempestivamente per consentire l'isolamento enterico del paziente.

È stato recentemente presentato il test immunoenzimatico C.DIFF CHEK (TechLab) per la glutammato deidrogenasi (GDH), enzima espresso da tutti i ceppi di $C$. difficile. Data la maggiore sensibilità $(92,7 \%)$, è stato proposto di usarlo come screening sulle feci di pazienti con probabile DAA, testando poi la tossina solo sui campioni positivi.

Scopo del nostro lavoro è stato verificare l'efficacia di tale proposta affiancando C.DIFF CHEK alle procedure di routine nel nostro laboratorio.

Metodi.

I campioni fecali di pazienti con sospetta DAA inviati al 
Servizio di Patologia Clinica dell'Ospedale di Desio (MI) nel periodo 18/03/05-13/06/05 sono stati sottoposti alle seguenti analisi: PREMIER TOXINS A\&B (Meridian), coltura su C.D. Selective Agar (Becton Dickinson) dopo selezione delle spore al calore, C.DIFF CHEK (TechLab).

\section{Risultati.}

Sono stati analizzati 194 campioni appartenenti a 98 soggetti. Dei 21 pazienti inizialmente tossina-positivi, 18 sono risultati C.DIFF CHEK-positivi, 3 negativi. Un soggetto tossina-positivo solo al secondo campione era fin dall'inizio C.DIFF CHEK-positivo. Dei 76 pazienti persistentemente tossina-negativi, 18 sono risultati C.DIFF CHEK-positivi.

Dei 194 campioni, 71 sono risultati positivi al C.DIFF CHEK, 43 alla coltura.

Conclusioni.

In base ai nostri risultati preliminari, l'uso di C.DIFF CHEK come screening non è consigliabile, poiché 3 pazienti su 22 con DAA da C.difficile sarebbero sfuggiti alla diagnosi. Riteniamo invece utile eseguire questo test, più rapido e sensibile dell'esame colturale, su campioni tossina-negativi per individuare tempestivamente casi comunque sospetti. 